\title{
Avaliação da demanda de cloro na oxidação de cianobactérias e relaç̧ão com a formação de trialometanos
}

\section{Estimate of chlorine demand of cyanobacteria oxidation and its relation with trihalomethanes formation}

Data de entrada: $15 / 12 / 2017$

Data de aprovação: 03/06/2019

Elton Santos Franco ${ }^{1 *}$ | Júlia Araújo Camargo ${ }^{1}$ | Núbia Aparecida de Aguilar ${ }^{1}$ | Ana Flávia de Assis Ferreira' | Valter Lúcio de Pádua ${ }^{2}$ | Jairo Lisboa Rodrigues' ${ }^{1}$ | Marcelo Libânio ${ }^{2}$ | Alessandra Giani²

DOI: https://doi.org/10.36659/dae.2020.072

ORCID ID

Franco ES (DD https://orcid.org/0000-0001-5296-4790 Camargo JA (D) https://orcid.org/0000-0003-4602-1010 Aguilar NA (D) https://orcid.org/0000-0001-5452-940X Ferreira AFA (D) https://orcid.org/0000-0002-5683-1917
Pádua VL (iD https://orcid.org/0000-0001-7972-1270

Rodrigues JL (D) https://orcid.org/0000-0001-6088-5232

Libânio M (D) https://orcid.org/0000-0002-0978-7474

Giani A (DD https://orcid.org/0000-0002-2524-8534

\section{Resumo}

Este trabalho teve como objetivo avaliar a demanda de cloro e a formação de trialometanos (TAMs) a partir da oxidação de células de Microcystis aeruginosa, espécie de cianobactéria amplamente encontrada nos mananciais brasileiros. $O$ estudo foi realizado em escala de laboratório avaliando 4 parâmetros: dose de cloro, tempo de contato, $\mathrm{pH}$ e temperatura, fatores que potencialmente influenciam a formação de subprodutos da desinfecção. Os TAMs foram determinados a partir de adaptação de método analítico de extração líquido líquido (ELL) em um cromatógrafo a gás acoplado à espectrometria de massas (CG-EM), que apresentou ótimo desempenho e economia quando comparado com outras metodologias. Os resultados demonstraram proporcionalidade entre a formação de TAMs e o aumento dos parâmetros analisados.

Palavras-chave: Trialometanos. Microcystis aeruginosa. CG-EM.

\section{Abstract}

This research had the objective to evaluate the chlorine demand and trihalomethanes (THMs) formation from the oxidation of Microcystis aeruginosa cells, species of cyanobacteria widely found in Brazilian waters. The study was performed in laboratory scale evaluating 4 parameters: chlorine doses, contact time, $\mathrm{pH}$ and temperature, factors that potentially influence the disinfection by-products formation. The THMs were determined from a liquid-liquid analytical method (LLE) adaptation in a gas chromatograph coupled to the mass spectrometry (GC-MS), which presented optimum performance and economy when compared to other methodologies. The results showed proportionality between the THM formation and the increase of the analyzed parameters.

Keywords: Trihalomethanes. Microcystis aeruginosa. GC-MS.

\footnotetext{
${ }^{1}$ Universidade Federal dos Vales do Jequitinhonha e Mucuri (UFVJM) - Teófilo Otoni - Minas Gerais - Brasil.

2 Universidade Federal de Minas Gerais (UFMG) - Belo Horizonte - Minas Gerais - Brasil.

* Autor correspondente: elton.santosaufvjm.edu.br.
} 


\section{INTRODUÇÃO}

O principal agente oxidante químico utilizado nas estações de tratamento de água (ETAs) na etapa de desinfecção é o cloro, devido ao seu baixo custo, praticidade de aplicação, residual persistente e efetividade na inativação de micro-organismos patogênicos quando comparado aos demais oxidantes. Apesar dos benefícios no processo de controle de patógenos, estudos demonstram que a utilização do cloro pode contribuir para a formação de subprodutos orgânicos halogenados (SOHs) indesejados, tais como os trialometanos (TAMs) e ácidos haloacéticos (AHAs), quando há presença de matéria orgânica natural (MON) na água (FLORENTIN et al., 2011; BUTIÃO, 2011; LIMA, 2014; CARDADOR et al., 2015).

Os TAMs constituem uma das primeiras famílias de $\mathrm{SOH}$ a serem descobertas na água e são a classe mais intensamente estudada. Apresentam um átomo de carbono em que três átomos de hi- drogênio são substituídos por diferentes halogênios como o bromo, o cloro e o iodo. Os mais frequentes na água, e por isso objetos de controle por meio de normas de qualidade da água cada vez mais restritivas, são: o triclorometano (TCM), o bromodiclorometano (BDCM), o dibromoclorometano (DBCM) e o tribromometano (TBM), cujas estruturas químicas são apresentadas na Fig. 1. O monitoramento desses compostos, também conhecidos como TAMs totais $\left(\right.$ TAM $\left._{\mathrm{T}}\right)$, é exigido pela Portaria de Consolidação n ${ }^{\circ} 5$ de 2017, anexo XX, do Ministério da Saúde, que limita o valor da soma dos mesmos a $100 \mu \mathrm{g} \cdot \mathrm{L}^{-1}$. Os TAMs são substâncias classificadas pela USEPA (1996) como classe B2 (provável carcinogênico para humanos, com evidências suficientes em animais) para o TCM, BDCM e TBM e classe $C$ (evidências limitadas de estudos em animais e dados inadequados ou inexistentes para humanos) no caso específico do DBCM.

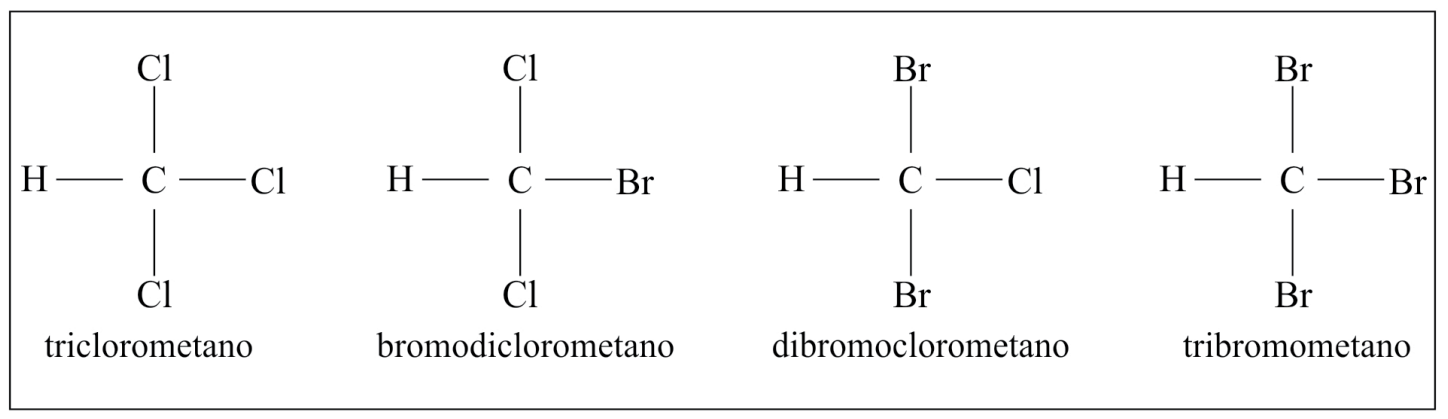

Figura 1 - Principais trialometanos formados no processo de oxidação em ETAs.

Vários são os fatores que podem influenciar a reação de formação de TAMs, destacando-se pH do meio, tempo de contato e temperatura, além da MON, considerada a principal precursora a ser convertida em TAMs na presença de cloro. As atividades antrópicas têm afetado diretamente os corpos d'água, pois os despejos de poluentes provenientes do aumento da produção de efluen- tes domésticos, agrícola e industrial têm alto teor de matéria orgânica e nutrientes. Essas têm sido uma das causas da proliferação excessiva de algas e cianobactérias potencialmente tóxicas em reservatórios e corpos hídricos usados para abastecimento público, propiciando o fenômeno conhecido como eutrofização. Também conhecido na literatura como floração ou bloom (concentrações 
superiores a 1,0 $010^{5}$ células. $\mathrm{mL}^{-1}$ ), o fenômeno tem se tornado frequente e é relevante uma vez que promove alterações nas características físico-químicas e na cadeia trófica dos ecossistemas aquáticos (MA et al., 2012; ZAMYADI et al., 2012; FUNASA, 2015).

De acordo com Zamyadi et al. (2013), a presença de cianobactérias pode ser representada pela presença de células e relacionadas com a matéria orgânica intracelular (MOI), a qual é liberada no ambiente aquático após lise celular e induzida pelo envelhecimento da população, constituídas principalmente por cianotoxinas, e matéria orgânica extracelular (MOE), que é resultante da atividade metabólica das células durante crescimento das fases exponenciais e estacionárias, substâncias como polissacarídeos, proteínas, lipídios e ácidos nucléicos, com contribuições significativas para a formação de SOHs.

Apesar de estudos apontarem algas verdes como importantes precursoras na formação de TAMs, vários pesquisadores destacam e relacionam gêneros de cianobactérias como parte importante na composição da matéria orgânica algogênica (MOA) e, também, na formação de TAMs, devido à sua predominância em eventos de eutrofização e relação com a demanda de cloro durante o processo de oxidação (ZAMYADI et al., 2012). No Brasil, destaca-se a espécie Microcystis aeruginosa, frequentemente associada a florações tóxicas (SANT'ANNA et al., 2008; ARAGÃO, 2011; CETESB, 2013; FUNASA, 2015), também mencionada pela literatura internacional (ISAACS et al., 2014; RZYMSKI e PONIEDZIAtEK, 2014; WILLIS et al., 2016; LEHMAN et al., 2017).

De modo geral, pode-se determinar os $\mathrm{SOHs}$ pela técnica de cromatografia. Entretanto, por estarem em baixas concentrações, necessitam de uma etapa de pré-concentração. Os mesmos podem ser isolados da matriz aquosa pela técnica de headspace (HS), purge and trap (PT) ou extração líquido-líquido (ELL), seguida pela determinação por cromatografia a gás por captura de elétrons (CG - DCE) ou cromatografia a gás acoplada à espectrometria de massas (CG-EM) (USEPA, 1995; NIKOLAOU et al., 2002; CULEA et al., 2006; PAVON et al., 2008).

Diante da relevância do contexto apresentado, o estudo tem como objetivo avaliar a influência da MOA, com foco em cianobactérias, na formação de TAMs, em condições distintas de $\mathrm{pH}$, temperatura e doses distintas de oxidante, utilizando-se espécies de cianobactérias Microcystis aeruginosa, que apresentam ampla distribuição geográfica no Brasil e em diversos países.

\section{METODOLOGIA}

\subsection{Curva de calibração para quantificação de TAMs}

As condições de extração para a determinação cromatográfica foram baseadas nas recomendações do método 551.1 da USEPA (USEPA, 1995). Soluções com os valores de concentração de 1 , $2,5,10,20,40,60,80,150$ e $200{\mu \mathrm{gL}^{-1}}^{-1}$ todas em triplicata, advindos de um padrão certificado de pureza cromatográfica de TAMs (TCM, BDCM, DBCM e TBM, $2000 \mu \mathrm{g} \cdot \mathrm{mL}^{-1}$ cada, 4M8140-U, TraceCERT ${ }^{\circledR}$ ), foram preparadas em $50 \mathrm{~mL}$ de água ultrapura. Para o procedimento de ELL, alíquotas de água isenta de compostos orgânicos voláteis e com concentração de $200 \mu \mathrm{g} \cdot \mathrm{L}^{-1}$ de TAMs foram coletadas nos volumes $5,10,20$, $30,40,50,60,80$ e $100 \mathrm{~mL}$ e inseridas em balões volumétricos, aos quais foi adicionado padrão interno fluorbenzeno na concentração correspondente ao volume contido nos balões volumétricos, $4 \mathrm{~mL}$ de MTBE, mantido em freezer a $4^{\circ} \mathrm{C}$, e aproximadamente 1,0 grama de sulfato de sódio anidro, agente secante da fase orgânica. $O$ frasco foi agitado vigorosamente por 5 minutos e imediatamente acondicionado em freezer $\mathrm{a}-4^{\circ} \mathrm{C}$. Duas fases bem definidas formaram-se: 
a fase orgânica superior, chamada de extrato, e a fase inferior, aquosa. A fase orgânica foi retirada e transferida para um frasco tipo vial de 2 $\mathrm{mL}$, que foi acondicionada em freezer $\mathrm{a}-4^{\circ} \mathrm{C}$ para conservação até a análise no CG-EM.

Para a identificação da presença dos TAMs, primeiramente foram realizadas extrações das espécies para obter a corrida cromatográfica do método utilizado, tendo como referência os valores monitorados por USEPA 551.1 (USEPA, 1995), Nikolaou et al. (2002), Culea et al. (2006). Definiu-se o modo de detecção TIC (total ion current, picos correspondentes a todas substâncias eluídas) e os tempos de retenção do modo SIR (selected ion recording, construído a partir do TIC, mas apenas usando fragmentos dos compostos $\mathrm{m} / \mathrm{z}$ ) para cada TAMs (TCM, BDCM, DBCM e TBM). Após a identificação das massas, por meio da relação massa/carga (m/z), procedeu-se com a otimização da rampa para a separação de cada espécie de TAMs e do padrão interno fluorbenzeno por meio do cromatógrafo.

Para as análises, foi utilizado um sistema de CG-EM, CLARUS 680 SQ 8PerkinElmer (USA). As condições otimizadas para o CG foram: $2 \mu \mathrm{L}$ de amostra injetada no modo splitless e separados por coluna capilar ZEBRON ZB - 5MS 30 $\mathrm{m}$ length $\times 0,25 \mathrm{~mm}$ diameter $\times 0,25 \mu \mathrm{m}$ film; fase $\mathrm{G} 27$ - 5\% fenil arileno e $95 \%$ dimetilpolisiloxano; injetor com temperatura de $200^{\circ} \mathrm{C}$; gás de arraste hélio de grau de pureza 99,9995\% com fluxo constante de $1,0 \mathrm{~mL}$.minuto ${ }^{-1}$; forno programado isotermicamente a $35^{\circ} \mathrm{C}$ por 1 minuto e, em seguida aumentada para $40^{\circ} \mathrm{C}$ a uma velocidade de $1^{\circ} \mathrm{C}$.minutos ${ }^{-1}$ durante $1 \mathrm{mi}-$ nuto, seguido de aquecimento de $30^{\circ} \mathrm{C}_{\text {. minuto }}{ }^{-1}$ até $200^{\circ} \mathrm{C}$. O EM foi usado no modo de impacto de elétrons $(\mathrm{EI}+)$. $\mathrm{O}$ tempo de corte do solvente foi a 3 minutos e velocidade de aquisição foi de 0,35 s.scan $^{-1}$, intervalo de leitura $\mathrm{m} / \mathrm{z}$ de 45 a 260 u.m.a. e modo de ionização de impacto eletrôni- co $70 \mathrm{eV}$. A temperatura da linha de transferência foi de $200^{\circ} \mathrm{C}$ e a temperatura do trap de $180^{\circ} \mathrm{C}$.

Por meio dos resultados, obtiveram-se regressões lineares $(Y=a X+b)$, onde $Y$ é $a$ área do pico obtida (descrito como sensibilidade ou intensidade) e X a concentração de cada TAM presente na amostra analisada no CG-EM. Para a análise das amostras pelo CG-EM, conforme orientado por Ribani et al. (2004), INMETRO (2016), ANVISA (2017) e tendo como base o estudo de Franco et al. (2018), que apresenta a validação de um método robusto de CG-EM, foram determinados os limites de detecção (LD) e quantificação (LQ). O LD representa a menor concentração da substância em exame que pode ser detectada, mas não necessariamente quantificada, e foi calculado com base na relação de 3,3 vezes o desvio padrão do branco dividido pelo coeficiente angular da curva analítica. O LQ representa a menor concentração da substância analisada que pode ser medida foi calculado pela relação de 10 vezes o desvio padrão do branco dividido pelo coeficiente angular da curva analítica.

\subsection{Manutenção, cultivo e contagens de células Microcystis aeruginosa}

A densidade de 1,0 $\times 10^{6}$ células. $\mathrm{mL}^{-1}$ foi definida por ser usual em mananciais de abastecimento eutrofizados (JARDIM et al., 2000; RODRIGUES, 2008; SILVA et al., 2013; REBOUÇAS, 2014; LEHMAN et al., 2017). Conforme orientação de Jacinavicius et al. (2013), para as cepas uniespecíficas de Microcystis aeruginosa, mantiveram-se as seguintes condições de cultivo: temperatura $24 \pm 2^{\circ} \mathrm{C}$, irradiância 40-50 $\mu \mathrm{mol}$ fótons $\mathrm{m}^{-2} \cdot \mathrm{s}^{-1}$ e fotoperíodo 14-10 horas claro-escuro, meio de cultivo ASM-1 (armazenado no freezer a $-4^{\circ} \mathrm{C}$ até ser diluído para uso), cultivadas em erlenmeyers replicados de 500 $\mathrm{mL}$ e $1 \mathrm{~L}$. Houve necessidade de ajustar o $\mathrm{pH}$ da solução a 7,4; adicionando $\mathrm{HCl}$ (ácido clorídrico) $1 \mathrm{M}$ para acidificar o meio e $\mathrm{NaOH}$ (hidróxido de sódio) $1 \mathrm{M}$ para elevar o pH. Para a contagem de células foi utilizada a câmera de Neubauer. 


\subsection{Avaliação da demanda de cloro para} definição das doses e dos principais fatores que influenciam a formação de TAMs

O oxidante utilizado no estudo foi o cloro gasoso. A real concentração de cloro da solução foi determinada por titulação, sendo o tiossulfato de sódio o titulante, e o amido e iodeto de potássio, os indicadores. 0 cloro foi utilizado imediatamente após o preparo para não haver perdas no processo por volatilização. Os valores das doses (D1 e D2) utilizadas para avaliação da formação de TAMs foram definidos por meio da adição de diferentes concentrações de cloro gasoso em soluções que continham Microcystis aeruginosa, conforme Fig. 2. Para a etapa de avaliação da demanda de cloro, foram utilizadas amostras com volume de $50 \mathrm{~mL}$ e concentrações de oxidante de 1,0; 1,5; 2,0; 2,5; 3,75; 5,0; 7,5 e 10,0 mg. $\mathrm{L}^{-1}$. O teor de cloro residual livre (CRL), após 30 minutos e 24 horas de contato, foi observado por método colorimétrico $\mathrm{HACH}$ Test Kit $\left(\mathrm{Cl}_{2}, 0\right.$ - 3,4 mg.L ${ }^{-1} \mathrm{Cl}_{2}$, modelo $\left.\mathrm{CN}-66\right)$ com adição de $\mathrm{N}$-dietil-para-fenilendiamina (DPD).

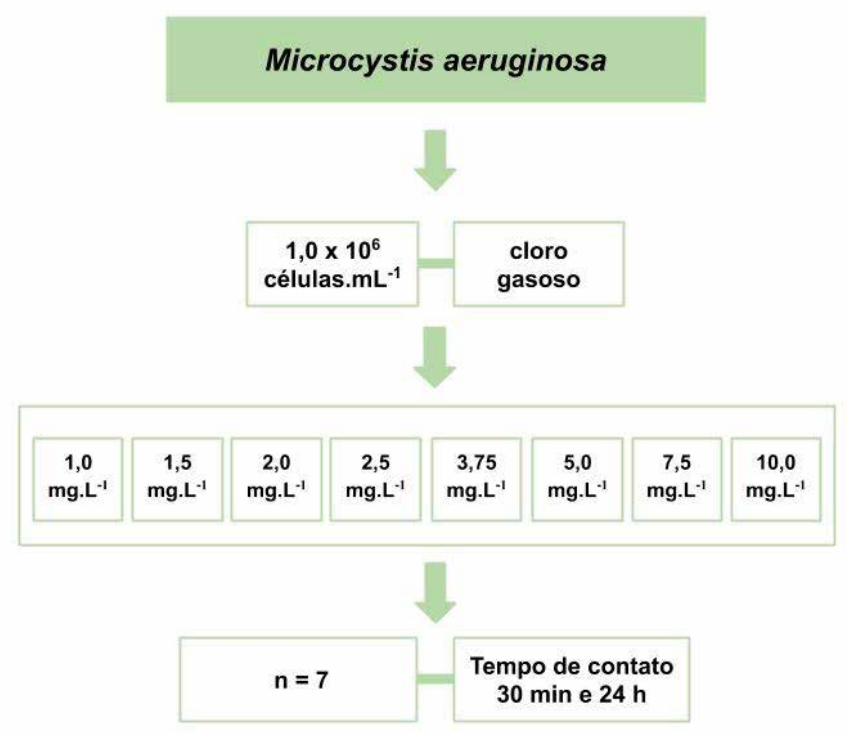

Figura 2 - Representação esquemática do método de ensaio para determinação das doses de cloro gasoso

Após os ensaios, as concentrações escolhidas foram as de 2,5 e 5,0 mg. $\mathrm{L}^{-1}$, visto que são as doses mais frequentemente utilizadas nas ETAs por apresentarem um residual de cloro em conformidade com o exigido pela portaria supracitada. Além das doses escolhidas, parâmetros como pH, temperatura e tempo de contato foram avaliados nas seguintes condições:

- $\mathrm{pH}: 6,5$ ( $\mathrm{pH}$ recomendado na etapa de coagulação) e 8,5 (valor recorrente em mananciais com florações de cianobactérias);
- Temperatura: $25^{\circ} \mathrm{C}$ e $30^{\circ} \mathrm{C}$ (temperaturas recorrentes no Brasil);

- Tempo de contato: 30 minutos (avaliação nos primeiros minutos de formação) e 24 horas (formação ao longo do sistema de distribuição).

A Fig. 3 apresenta o fluxograma do procedimento utilizado para realização dos ensaios de formação de TAMs, utilizando a espécie. 


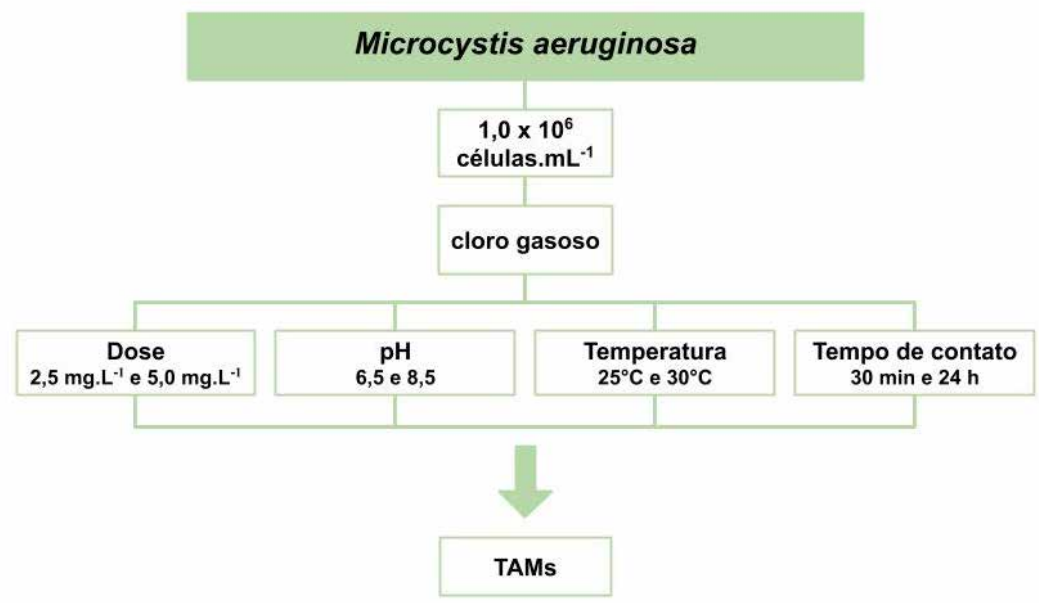

Figura 3 - Representação esquemática do método utilizado nos ensaios de formação de TAMs

\section{RESULTADOS E DISCUSSÃO}

\subsection{Microscopia}

No intuito de verificar contaminações de outros micro-organismos, imagens foram obtidas por meio do microscópio Nikon Eclipse E200 e proces- sadas pelo software ISCapture V3.6. As imagens obtidas com aumentos de 100X e 400X, apresentadas na Fig. 4, não mostraram presença de outros micro-organismos, descartando-se, então, a possibilidade de contaminação da amostra.

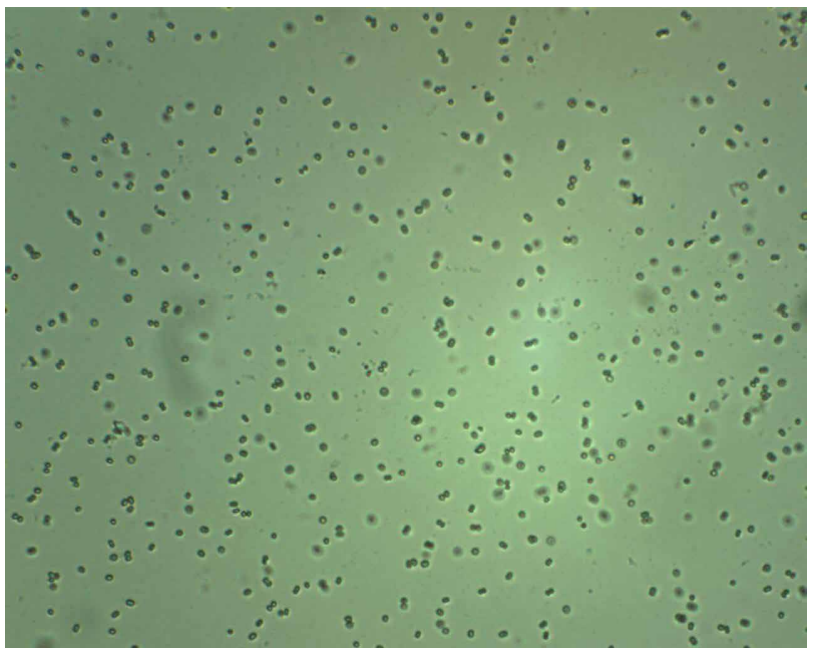

(a)

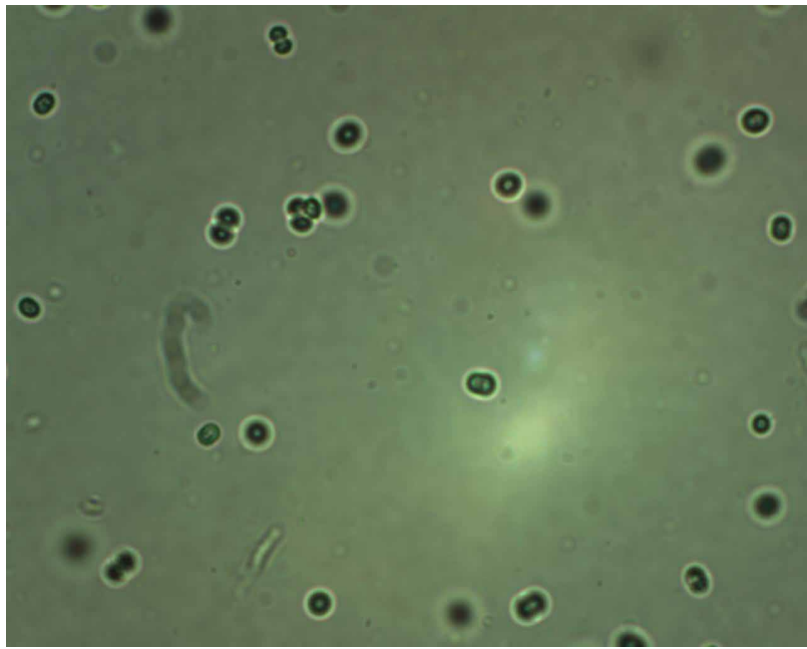

(b)

Figura 4 - Imagens ampliadas 100X (A) e 400X (B) de células de Microcystis aeruginosa presentes nas amostras analisadas

\subsection{Corridas cromatográficas}

O TCM foi o primeiro composto detectado, por apresentar menor massa molar, ao ponto que o TBM foi o último dos compostos, por apresen- tar a maior massa em relação aos outros TAMs. A sequência das espécies apresentadas é justificada pelas características distintas de cada composto. A Fig. 5 apresenta um dos cromatogramas obtidos. 


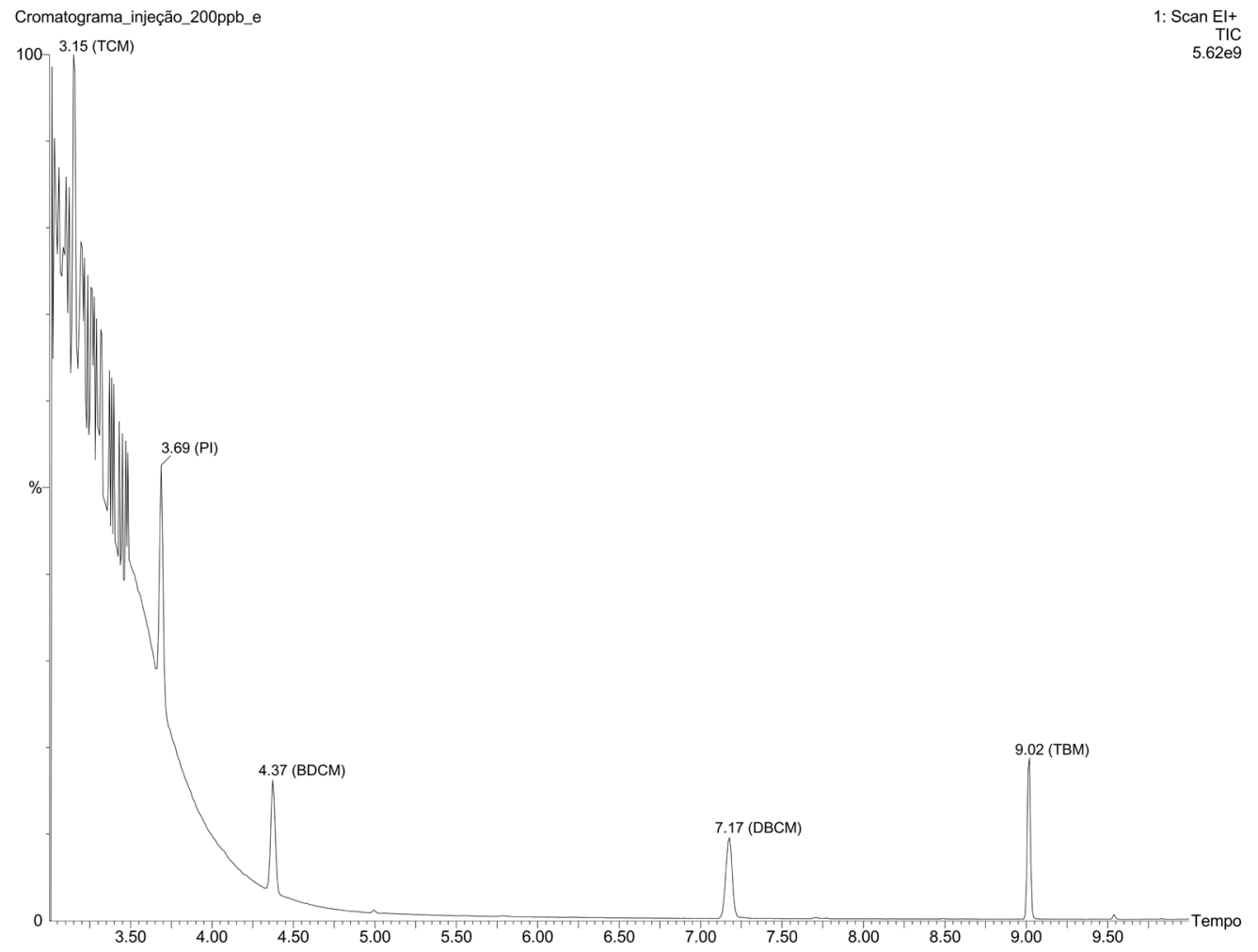

Figura 5 - Cromatograma modo TIC dos TAMs obtido nas condições cromatográficas otimizadas, com presença do padrão interno fluorbenzeno, obtido por injeção a partir de uma extração na concentração $200 \mu \mathrm{g} \cdot \mathrm{L}^{-1}$

\subsection{Curvas Analíticas}

Conforme Tabela 1, para todas as espécies de TAMs, obtiveram-se coeficientes de correlação (R) superiores a 0,99. A Anvisa (2017) recomenda valores superiores a 0,99, e o Inmetro (2016), um mínimo de 0,9.

Tabela 1 - Curvas de calibração dos trialometanos.

\begin{tabular}{|c|c|c|}
\hline TAMs & Curva de calibração* & $\begin{array}{c}\text { Coeficiente de } \\
\text { correlação (R) }\end{array}$ \\
\hline TCM & $Y=1,0229+0,6075 \times X$ & 0,9991 \\
\hline BDCM & $Y=-1,8411+0,6974 \times X$ & 0,9969 \\
\hline DBCM & $Y=-3,4270+0,7185 \times X$ & 0,9951 \\
\hline TBM & $Y=-2,0128+0,3767 \times X$ & 0,9950 \\
\hline
\end{tabular}

*Yé a área do pico obtida e Xé a concentração de cada TAM.

A Tabela 2 apresenta os valores obtidos para o LD e LQ com base nas relações apresentadas no item 2.1. É importante ressaltar que o Inmetro (2016) e a Anvisa (2017) não estabelecem um valor mínimo para tais parâmetros, pois os mesmos podem variar em função do tipo de amostra.

Tabela 2 - Limites de detecção (LD) e quantificação (LQ) obtidos para os trialometanos.

\begin{tabular}{|c|c|c|c|c|}
\hline Parâmetro & TCM & BDCM & DBCM & TBM \\
\hline Limite de detecção (LD) $\left(\mu \mathrm{g} . \mathrm{L}^{-1}\right)$ & 0,13 & 0,02 & 0,02 & 0,01 \\
\hline $\begin{array}{c}\text { Limite de quantificação (LQ) } \\
\left(\mu \mathrm{g} \cdot \mathrm{L}^{-1}\right)\end{array}$ & 0,40 & 0,07 & 0,07 & 0,05 \\
\hline
\end{tabular}

\subsection{Ensaios de demanda de cloro após cloração de células Microcystis aeruginosa}

As Tabelas 3 e 4 a seguir apresentam os resultados de cloro residual livre, em mg. $\mathrm{L}^{-1}$, obtidos para cada ensaio realizado e suas respectivas doses e tempo de contato. Tais concentrações foram escolhidas para analisar o consumo de cloro para doses abaixo e acima das concentrações de cloro usualmente utilizadas (2,5 e 5,0 mg. $\mathrm{L}^{-1}$ ). 
Tabela 3 - Concentração de cloro residual livre $\left(\mathrm{mg}^{\mathrm{L}} \mathrm{L}^{-1}\right)$ obtida para 30 minutos de contato, na densidade de $1,0 \times 10^{6}$ células. $\mathrm{mL}^{-1}$

\begin{tabular}{|c|c|c|c|c|c|c|c|c|c|c|}
\hline $\begin{array}{c}\text { cloro } \\
\text { gasoso } \\
\left.\text { (mg. } L^{-1}\right)\end{array}$ & $\begin{array}{c}\text { Tempo de } \\
\text { contato }\end{array}$ & Ensaio 1 & Ensaio 2 & Ensaio 3 & Ensaio 4 & Ensaio 5 & Ensaio 6 & Ensaio 7 & Média & $\begin{array}{l}\text { Desvio } \\
\text { padrão }\end{array}$ \\
\hline 1,0 & \multirow{8}{*}{30 minutos } & 0,10 & 0,10 & 0,10 & 0,10 & 0,10 & 0,10 & 0,10 & 0,10 & 0,00 \\
\hline 1,5 & & 0,10 & 0,10 & 0,10 & 0,10 & 0,10 & 0,10 & 0,10 & 0,10 & 0,00 \\
\hline 2,0 & & 0,10 & 0,10 & 0,10 & 0,10 & 0,10 & 0,10 & 0,15 & 0,11 & 0,02 \\
\hline 2,5 & & 0,10 & 0,10 & 0,10 & 0,10 & 0,10 & 0,10 & 0,20 & 0,11 & 0,03 \\
\hline 3,75 & & 0,10 & 0,20 & 0,10 & 0,15 & 0,15 & 0,15 & 0,18 & 0,15 & 0,03 \\
\hline 5,0 & & 0,10 & 0,20 & 0,10 & 0,20 & 0,20 & 0,20 & 0,18 & 0,17 & 0,04 \\
\hline 7,5 & & 0,10 & 0,50 & 0,20 & 0,60 & 0,80 & 0,60 & 0,60 & 0,49 & 0,23 \\
\hline 10,0 & & 0,30 & 0,50 & 0,30 & 1,80 & 1,80 & 1,80 & 1,80 & 1,19 & 0,71 \\
\hline
\end{tabular}

Tabela 4 - Concentração de cloro residual livre $\left(\mathrm{mg} \cdot \mathrm{L}^{-1}\right)$ obtida para

24 horas de contato, na densidade de $1,0 \times 10^{6}$ células. $\mathrm{mL}^{-1}$

\begin{tabular}{|c|c|c|c|c|c|c|c|c|c|c|}
\hline $\begin{array}{c}\text { cloro } \\
\text { gasoso } \\
\left(\mathrm{mg} \cdot \mathrm{L}^{-1}\right)\end{array}$ & $\begin{array}{c}\text { Tempo de } \\
\text { contato }\end{array}$ & Ensaio 1 & Ensaio 2 & Ensaio 3 & Ensaio 4 & Ensaio 5 & Ensaio 6 & Ensaio 7 & Média & $\begin{array}{l}\text { Desvio } \\
\text { padrão }\end{array}$ \\
\hline 1 & \multirow{8}{*}{24 horas } & 0,10 & 0,10 & 0,10 & 0,10 & 0,10 & 0,10 & 0,10 & 0,10 & 0,00 \\
\hline 1,5 & & 0,10 & 0,10 & 0,10 & 0,10 & 0,10 & 0,10 & 0,10 & 0,10 & 0,00 \\
\hline 2 & & 0,10 & 0,10 & 0,10 & 0,10 & 0,10 & 0,10 & 0,10 & 0,10 & 0,00 \\
\hline 2,5 & & 0,10 & 0,10 & 0,10 & 0,10 & 0,10 & 0,10 & 0,10 & 0,10 & 0,00 \\
\hline 3,75 & & 0,10 & 0,10 & 0,10 & 0,10 & 0,10 & 0,10 & 0,10 & 0,10 & 0,00 \\
\hline 5,0 & & 0,10 & 0,10 & 0,10 & 0,20 & 0,20 & 0,20 & 0,10 & 0,14 & 0,05 \\
\hline 7,5 & & 0,10 & 0,10 & 0,10 & 0,20 & 0,20 & 0,20 & 0,20 & 0,16 & 0,05 \\
\hline 10,0 & & 0,20 & 0,10 & 0,10 & 0,20 & 0,20 & 0,20 & 0,20 & 0,17 & 0,05 \\
\hline
\end{tabular}

Analisando-se os resultados de cloro residual livre obtidos para cada ensaio e tempos de contato, pode-se afirmar que houve um residual menor que $20 \%$ do valor das respectivas doses aplicadas, o que demonstra um elevado consumo de oxidante. Como pode ser observado na Tabela 3, as doses 7,5 e 10,0 mg. $\mathrm{L}^{-1}$ apresentaram maiores média e desvio padrão, o que pode ser explicado pelo maior residual de cloro obtido em alguns ensaios para essas doses do que para as demais. 0 maior residual obtido provavelmente se deve ao fato de o meio apresentar menor concentração de MOE e MOI, não demandando tanto oxidante para oxidação da matéria orgânica presente. $\mathrm{O}$ alto consumo de cloro e, consequentemente, baixo CRL pode estar relacionado ao fato de que, segundo Fang et al. (2010), a lise celular pode levar a uma liberação de toxinas ligadas à célula, carbono orgânico dissolvido (COD) e nitrogênio orgânico, que apresenta demanda significativa de cloro. Culturas de cianobactérias contêm uma composição heterogênea de compostos, como a MOE e MOI e, segundo $\mathrm{Ma}$ et al. (2012), após o cloro adentrar nas células, reage com diferentes materiais, tais como membranas celulares e substâncias intracelulares, aumentando a demanda.

Zamyadi et al. (2012) destacam que baixas doses podem resultar em rápida liberação de toxinas e subsequente oxidação das mesmas, ocasionando maior consumo de cloro. Observaram ainda, por meio de modelagens matemáticas, que a oxidação dessas toxinas, total ( $\mathrm{k}$ total) ou dissolvida ( $\mathrm{k}$ dissolvido), ocorre mais rapidamente do que a lise celular ( $\mathrm{k}$ lise), sugerindo que as toxinas liberadas ligadas às células reajam mais rapidamente com o cloro que o restan- 
te de células danificadas e outras substâncias orgânicas dissolvidas. Ademais, demonstraram que, além da liberação de toxina, a oxidação por cloro promoveu a perda da integridade das células, o que pode explicar a presença de células imóveis e menores que o tamanho real nas amostras cloradas no atual trabalho.

\subsection{Análise de formação de trialometanos após cloração de Microcystis aeruginosa}

Dentre os TAMs analisados, o TCM foi a única espécie presente em todas as amostras, corroborando com a maioria dos estudos na literatura, e as concentrações encontradas estão apresentadas na Tabela 5. 0 BDCM, DBCM e TBM não foram detectados.

Tabela 5 - Concentração de TAMs após cloração da Microcystis aeruginosa

\begin{tabular}{|c|c|c|c|c|c|c|}
\hline $\begin{array}{l}\text { Oxidante } \\
\text { químico }\end{array}$ & Dose $\left(\mathrm{mg} \cdot \mathrm{L}^{-1}\right)$ & Temperatura & $\mathrm{pH}$ & $\begin{array}{l}\text { Tempo de } \\
\text { contato }\end{array}$ & TCM $\left(\mu \mathrm{g} \cdot \mathrm{L}^{-1}\right)$ & TAMT $\left(\mu \mathrm{g} \cdot \mathrm{L}^{-1}\right)$ \\
\hline \multirow{16}{*}{$\begin{array}{l}\text { cloro } \\
\text { gasoso }\end{array}$} & \multirow{8}{*}{2,5} & $25^{\circ} \mathrm{C}$ & 6,5 & \multirow{4}{*}{30 minutos } & 2,16 & 2,16 \\
\hline & & $25^{\circ} \mathrm{C}$ & 8,5 & & 3,17 & 3,17 \\
\hline & & $30^{\circ} \mathrm{C}$ & 6,5 & & 2,98 & 2,98 \\
\hline & & $30^{\circ} \mathrm{C}$ & 8,5 & & 4,23 & 4,23 \\
\hline & & $25^{\circ} \mathrm{C}$ & 6,5 & \multirow{4}{*}{24 horas } & 2,91 & 2,91 \\
\hline & & $25^{\circ} \mathrm{C}$ & 8,5 & & 4,19 & 4,19 \\
\hline & & $30^{\circ} \mathrm{C}$ & 6,5 & & 17,93 & 17,93 \\
\hline & & $30^{\circ} \mathrm{C}$ & 8,5 & & 19,78 & 19,78 \\
\hline & \multirow{8}{*}{5,0} & $25^{\circ} \mathrm{C}$ & 6,5 & \multirow{4}{*}{30 minutos } & 2,66 & 2,66 \\
\hline & & $25^{\circ} \mathrm{C}$ & 8,5 & & 3,64 & 3,64 \\
\hline & & $30^{\circ} \mathrm{C}$ & 6,5 & & 3,45 & 3,45 \\
\hline & & $30^{\circ} \mathrm{C}$ & 8,5 & & 5,39 & 5,39 \\
\hline & & $25^{\circ} \mathrm{C}$ & 6,5 & \multirow{4}{*}{24 horas } & 5,56 & 5,56 \\
\hline & & $25^{\circ} \mathrm{C}$ & 8,5 & & 7,13 & 7,13 \\
\hline & & $30^{\circ} \mathrm{C}$ & 6,5 & & 18,71 & 18,71 \\
\hline & & $30^{\circ} \mathrm{C}$ & 8,5 & & 31,22 & 31,22 \\
\hline
\end{tabular}

Observou-se que em todas as amostras com $\mathrm{pH}$ 8,5, para o oxidante e ambas doses, a concentração de TAMs gerada foi maior do que aquelas com pH 6,5, comparando as amostras de mesma temperatura. Esses resultados estão de acordo com estudos da literatura (LATIF,1991; MEYER, 1994; SINGER, 1999; HUA e RECKOW, 2008), que, avaliando a formação de TAMs em situações distintas de $\mathrm{pH}$, observaram um aumento na concentração desses compostos em valores mais básicos. O estudo de Hespanhol et al. (1982) mostrou um aumento na formação de TAMs de até três vezes maior em valores de $\mathrm{pH}$ mais básicos. Os resultados obtidos para as temperaturas estudadas, nas duas doses de oxidantes utilizadas, foram ao encontro da maioria dos estudos observados (BORGES, 2003; FUNASA, 2007; SUN et al., 2009; LEE et al., 2013), visto que a maior parte das amostras apresentou valores maiores de TAMs quando incubadas a $30^{\circ} \mathrm{C}$ do que a $25^{\circ} \mathrm{C}$. Para o parâmetro tempo de contato, de acordo com a literatura, há uma alta correlação com a formação, o que pode ter influenciado os resultados, visto que para um maior tempo de contato houve maior formação de TAMs. Diante dos resultados obtidos, pode-se afirmar que para maiores valores de cada parâmetro houve uma maior formação da espécie TCM, que foi a única formada neste estudo, demonstrando que todos os parâmetros analisados influenciam diretamente na formação desse subproduto.

O estudo de Anágua (2011), que utilizou água sintética com ausência bromo em sua composição e hipoclorito de sódio como oxidante, apre- 
sentou a formação das quatro espécies de TAMs nas seguintes concentrações ( 2 horas de contato): $6,2 \mu \mathrm{g} \cdot \mathrm{L}^{-1}$, para TCM; $2,0 \mu \mathrm{g} \cdot \mathrm{L}^{-1}$, para BDCM; $1,6 \mu \mathrm{g} \cdot \mathrm{L}^{-1}$, para DBCM; 0,9 $\mu \mathrm{g} \cdot \mathrm{L}^{-1}$, para TBM. Segundo a autora, a presença de íons brometo no meio aumenta a tendência para a formação de espécies bromadas de TAMs, ou seja, a presença dos íons não garante obrigatoriamente a formação de tais espécies. Sendo assim, a não detecção de espécies bromadas no presente estudo, que também utilizou meio de cultivo ausente de bromo, deve-se, provavelmente, à baixa concentração dos compostos ou ausência de íons brometo.

Ainda, o estudo de Sun et al. (2009), que analisou águas residuais, apresenta uma relação entre a temperatura e a formação de TAMs. Para temperaturas mais baixas $\left(4^{\circ} \mathrm{C}\right)$, os autores obtiveram uma maior formação das espécies bromadas do que para temperaturas mais elevadas $\left(25^{\circ} \mathrm{C}\right.$ e $30^{\circ} \mathrm{C}$, também usadas no presente estudo). Para um maior tempo de contato, os autores obtiveram maior concentração das espécies bromadas do que para menor tempo, o que contradiz os resultados obtidos no presente trabalho, onde a diferença significativa do tempo de contato, conforme Tabela 5, não promoveu maior formação das espécies em questão de modo que pudessem ser detectadas pela instrumentação. Ao analisar os resultados obtidos pelos autores para o parâmetro $\mathrm{pH}$, a formação de TCM para $\mathrm{pH} 6$ e 7 foi significativamente mais elevada do que BDCM e DBCM. Para doses mais elevadas de cloro gasoso (40 mg. $\mathrm{L}^{-1}$ ), o estudo dos autores apresentou maior concentração de TCM e formação de BDCM e DBCM se comparado com as menores (5 mg. $\left.\mathrm{L}^{-1}\right)$ e, mesmo havendo a formação de espécies bromadas, o TCM foi a espécie dominante, representando mais de $71 \%$ da concentração total de TAMs formados.
Por fim, é importante ressaltar que os resultados obtidos neste estudo estão relacionados exclusivamente com as cianobactérias cloradas, cujas células são apresentadas na Fig. 4, e seus metabólitos, já que foram cultivadas em água ultrapura, apenas com o meio ASM-1 e sem contaminantes externos. A MOE e MOI podem contribuir para a formação dos $\mathrm{SOH}$, como os TAMs, devido à reação da matéria orgânica com cloro. Sendo assim, como não foi realizada a separação entre as células e MOE, as concentrações de TAMs formados podem também estar relacionados a esses compostos (HUREIKI et al.,1994; BOND et al., 2009). Além disso, os resultados mostram que o COD ligado à célula de cianobactérias pode ser fonte significativa de precursores de $\mathrm{SOHs}$ (NGUYEN et al, 2005; HUANG et al., 2009; YANG et al., 2011).

\section{CONCLUSÕES}

A quantificação dos TAMs por ELL-CG-EM foi possível, com excelentes resultados advindos de adaptações da USEPA 551.1 (USEPA, 1995) e Standard Methods for the Examination of Water and Wastewater (APHA, 2012). Ainda, a ELL apresentou eficácia, podendo-se dispensar métodos de extração mais onerosos, como headspace e purge and trap. Os ensaios de demanda de cloro gasoso para oxidação da Microcystis aeruginosa demonstraram altos valores de consumo e baixos índices de $\mathrm{CRL}$, possivelmente justificados pela reação de células MOI e MOE com o oxidante. Dos ensaios realizados, aproximadamente $28 \%$ das amostras apresentaram CRL em conformidade com o preconizado pela Portaria da Consolidação. A cloração das espécies na concentração $1,0 \times 10^{6}$ células. $\mathrm{mL}^{-1}$ propiciou a formação apenas de TCM, sendo as outras espécies em concentrações abaixo do LD ou não detectadas, mesmo cloradas em situações distintas de $\mathrm{pH}$, temperatura e dose do oxidante. Ainda pôde-se 
observar a importância dos parâmetros analisados na formação de TAMs, visto que os mesmos exercem influência direta na formação de TAMs, pois quanto maior o valor de cada parâmetro, maior foi a formação de subproduto.

\section{AGRADECIMENTOS}

Os autores agradecem à Fundação de Amparo à Pesquisa do Estado de Minas Gerais (FAPEMIG), as bolsas de iniciação científica vinculadas a pesquisa; e à Fundação Nacional de Saúde (FUNASA), pelo o apoio financeiro.

\section{CONTRIBUIÇÃO DOS AUTORES}

Todos os autores contribuíram de forma igualitária.

\section{REFERÊNCIAS}

ANVISA. Agência Nacional de Vigilância Sanitária, Resolução RE no 899. Guia para validação de métodos analíticos e bioanalíticos. Brasil, 2017. Disponível em <http://redsang.ial.sp.gov.br/ site/docs_leis/vm/vm1.pdf> Acesso em 14 setembro de 2017.

ANÁGUA, T. A. S. Trihalometanos como subprodutos da cloração. 2011. 73 f. Dissertação (Mestrado em Química Industrial) - Universidade da Beira Interior, Covilhã, Portugal, 2011.

APHA. AMERICAN PUBLIC HEALTH ASSOCIATION. Standard Methods for the Examination of Water and Wastewater. 22 ed., 1496 f., 2012.

ARAGÃO, N. K. C. V. Taxonomia, distribuição e quantificação de populações de cianobactérias em reservatórios do estado de Pernambuco (Nordeste do Brasil). 2011. 159 f. Dissertação (Mestrado em Botânica) - Departamento de Biologia, Universidade Federal Rural de Pernambuco, PE, 2011.

BRASIL. Ministério da Saúde. Portaria da Consolidação $n^{\circ} 5$, de 28 de setembro de 2017. Consolidação das normas sobre as ações e os serviços de saúde do Sistema Único de Saúde. Diário Oficial da União, 2017.

BOND, T.; HENRIET, O.; GOSLAN, E. H.; PARSONS, S. A.; JEFFERSON, B. Disinfection byproduct formation and fractionation behavior of natural organic matter surrogates. Environmental Science and Technology, v. 43, n. 15, p. 5982-5989, 2009.
BORGES, J. T. A utilização da técnica MIMS na determinação de trialometanos em águas de abastecimento e a influência do íon brometo, da amônia e de algas na formação desses compostos. 2003. 171 f. Tese de (Doutorado em Engenharia Civil) Área de concentração Saneamento e Ambiente - Faculdade de Engenharia Civil, Universidade Estadual de Campinas, Campinas, 2003.

BUTIÃO, D. F. Formação de subprodutos orgânicos halogenados da desinfecção em águas de piscinas. 2011. 78 f. Dissertação (Mestrado em Tecnologia Ambiental). Universidade de Ribeirão Preto, Ribeirão Preto, SP, 2011.

CARDADOR, M. J.; SALGUERO, J. F.; GALLEGO, M. Simultaneous quantification of trihalomethanes and haloacetic acids in cheese by on-line static headspace gas chromatography-mass spectrometry. Journal of Chromatography A, v. 1408, p. 22-29, 2015. https://doi.org/10.1016/j.chroma.2015.07.007

CETESB. Manual de Cianobactérias Platônicas: Legislação, Orientações para o Monitoramento e Aspectos Ambientais. São Paulo: CETESB, 2013.

CULEA, M.; COZAR, O.; RISTOIU, D. Methods validation for the determination of trihalomethanes in drinking water. Journal of Mass Spectrometry, v. 41, n. 12, p. 1594-1597, 2006. https:// doi.org/10.1002/jms.1149

FANG, J.; YANG, X.; MA, J.; SHANG, C.; ZHAO, Q. Characterization of algal organic matter and formation of DBPs from chlor (am) ination. Water Research, v. 44, p. 5897-5906, 2010. https://doi. org/10.1016/j.watres.2010.07.009

FLORENTIN, A.; HAUTEMANIÈRE, A.; HARTEMANN, P. Health effects of disinfection by-products in chlorinated swimming pools. International Journal of Hygiene and Environmental Health, v. 214, p. 461- 469, 2011. https://doi.org/10.1016/j.ijheh.2011.07.012

FRANCO, E. S.; PÁDUA, V. L.; GIANI, A.; RODRÍGUEZ, M.; SILVA, D. F.; FERREIRA, A. F. A; JÚNIOR, I. C. S.; PEREIRA, M. C.; RODRIGUES, J. L. Validation of a robust LLE-GC-MS method for determination of trihalomethanes in environmental samples. Environmental Monitoring and Assessment, v. 190, n. 473, 2018. https://doi. org/10.1007/s10661-018-6835-8

FUNDAÇÃO NACIONAL DE SAÚDE (FUNASA). Cianobactérias/ cianotoxinas: procedimentos de coleta, preservação e análise. Ministério da Saúde, Secretaria de Vigilância em Saúde, Departamento de Vigilância em Saúde Ambiental e Saúde do Trabalhador. - Brasília: Ministério da Saúde, 106 p. 2015.

FUNDAÇÃO NACIONAL DE SAÚDE (FUNASA). Potenciais fatores de risco à saúde decorrentes da presença de subprodutos de cloração na água utilizada para consumo humano. Brasília: Ministério da Saúde. 126 p. 2007.

HESPANHOL, I.; AZEVEDO NETO, J. M.; BOTELHO, M. H. C. Usos do cloro na engenharia sanitária e ambiental; novas tecnologias 
de aplicação e quantificação dos impactos associados. Revista DAE, v. 42, p. 61-71, 1982.

HUA, G.; RECKHOW, D. A. DBP formation during chlorination and chloramination: Effect of reaction time, $\mathrm{pH}$, dosage, and temperature. Journal American Water Works Association, v. 100, n. 8, p. 82, 2008. https://doi.org/10.1002/j.1551-8833.2008. tb09702.x

HUANG, J.; GRAHAM, N.; TEMPLETON, M. R.; ZHANG, Y.; COLLINS, C.; NIEUWENHUIJSEN, M. A comparison of the role of two blue-green algae in THM and HAA formation. Water Research, $v$. 43, n. 12, p.3009-3018, 2009. https://doi.org/10.1016/S00456535(00)00210-1

HUREIKI, L.; CROUE, J. P.; LEGUBE B. Chlorination studies of free and combined aminoacids. Water Research, v. 28, n. 12, 1994. https://doi.org/10.1016/0043-1354(94)90070-1

ISAACS, J. D.; STRANGMAN, W. K.; BARBERA, A. E.; MALLIN, M. A.; MCLVER, M. R.; WRIGHT, J. L. C. Microcystins and Two New Micropeptin Cyanopeptides Produced by Unprecedented Microcystis aeruginosa Blooms in North Carolina's Cape Fear River . Harmful Algae, v. 31, p. 82-86, 2014. https://doi.org/10.1016/j. hal.2013.09.010

INSTITUTO NACIONAL DE METROLOGIA, NORMALIZAÇÃO E QUALIDADE INDUSTRIAL (INMETRO). Orientação sobre Validação de Métodos Analíticos: Documento de caráter orientativo (DOQ-CGCRE-008). Revisão n 5, 31 p., ago. 2016.

JACINAVICIUS, F. R.; JÚNIOR. W, A, G.; AZEVEDO, M, T, P.; SANT'ANNA, C, L. Manual para Cultivo de Cianobactérias. 2013. Disponível em: <http://botanica.sp.gov.br/files/2013/09/ virtuais_4cianobact\%C3\%A9rias.pdf. > Acesso em 9 de setembro de 2015 .

JARDIM, F. A.; MACHADO, J. N. A.; SCHEMBRI, M. C. A. C.; AZEVEDO, S. M. F. O.; VON SPERLING, E. A experiência da COPASA no monitoramento, detecção e adoção de medidas mitigadoras para as cianobactérias tóxicas em estações de tratamento de água - Minas Gerais - Brasil. In:XXVII CONGRESSO INTERAMERICANO DE ENGENHARIA SANITÁRIA E AMBIENTAL. Porto Alegre, RS, 2000. Anais...

LATIF, N., 1991. Trihalomethane compounds in the drinking water of Kuwait: a survey from source to consumer. A Thesis submitted for the degree of Doctor of Philosophy, Brunel University. 1991. Disponível em < http://bura.brunel.ac.uk/handle/2438/4973> Acesso em 12 de janeiro de 2017.

LEE, J.; KIM, E.; ROH, B.; EOM, S.; ZOH, K. Occurrence of disinfection by-products in tap water distribution systems and their associated health risk. Environmental Monitoring and Assessment, v. 185, p. 7675-7691, 2013. https://doi.org/10.1007/ s10661-013-3127-1

LEHMAN, P. W.; KUROBE, T.; LESMEISTER, S.; BAXA, D.; TUNG, A.; TEH, S. J. Impacts of the 2014 Severe Drought on the Mi- crocystis Bloom in San Francisco Estuary (em inglês). Harmful Algae, v. 63, n. 12, p. 94-108, 2017. https://doi.org/10.1016/j. hal.2017.01.011

LIMA, G. M. Desenvolvimento de correlação da formação de ácidos haloacéticos em água contendo substância húmica oxidada com cloro. 2014. 109 f. Dissertação (Mestrado Profissionalizante em Tecnologia Ambiental) - Centro de Ciências Exatas, Naturais e Tecnologias da Universidade de Ribeirão Preto, Ribeirão Preto, 2014.

MA, M.; LIU, R.; LIU, H.; QU, J.; JEFFERSON, W. Effects and mechanisms of pre-chlorination on Microcystis aeruginosa removal by alum coagulation: Significance of the released intracellular organic matter. Separation and Purification Technology, v. 86, p. 19-25, 2012. https://doi.org/10.1016/j.seppur.2011.10.015

MEYER, S.T. O Uso de cloro na desinfecção de águas, a formação de trialometanos e os riscos potenciais à saúde pública. Caderno de Saúde Pública, v. 10, n. 1, p. 99-110, 1994. https://doi. org/10.1590/S0102-311X1994000100011

NGUYEN, M.L.; WESTERHOFF, P.; BAKER, L.; HU, Q.; ESPARZA-SOTO, M.; SOMMERFELD, M. Characteristics and reactivity of alga e produced dissolved organic carbon. Journal of Environmental Engineering, v.131, n. 11, p. 1574-1582, 2005. https://doi. org/10.1061/(ASCE)0733-9372(2005)131:11(1574)

NIKOLAOU, A. D.; LEKKAS, T. D.; GOLFINOPOULOS, S. K.; KOSTOPOULOU M. N. Application of different analytical methods for determination of volatile chlorination by-products in drinking water. Talanta, v. 56, p. 717-726, 2002. https://doi.org/10.1016/ s0039-9140(01)00613-0

PAVON, J. L. P.; MARTIN, S. H.; PINTO, C. G.; CORDERO, B. M. Determination of trihalomethanes in water samples: a review. Analytica Chimica Acta, v. 629, p. 6-23, 2008. https://doi.org/10.1016/j.aca.2008.09.042

REBOUÇAS, P. M. Desempenho de alevinos masculinizados de tilápia do Nilo (Oreochromis niloticus) produzidos em duas estações do ano em tanques de diferentes cores. 2014. 148 f. Dissertação (Mestrado em Engenharia Agrícola). Universidade Federal do Ceará. Fortaleza, CE, 2014.

RIBANI, M.; BOTTOLI, C.B.G.; COLLINS, C.H.; JARDIN, I.C.S.F.; MELO, L.F.C. Validation for chromatographic and electrophoretic methods. Química Nova, v. 27, n. 5, p. 771-780, 2004. https:// doi.org/10.1590/S0100-40422004000500017

RODRIGUES, L. L. Biodiversidade de cianobactérias e algas das represas Billings (Braço Taquacetuba), e Guarapiranga, SP, Brasil. 205 f. Dissertação. (Mestrado em Biociências, Ecologia) Universidade de São Paulo. São Paulo. São Paulo, 2008.

RZYMSKI, P.; PONIEDZIALEK, B. In Search of Environmental Role of Cylindropspermopsin: A Review on Global Distribution and Ecology of its Producers. Water Research, v. 66, p. 320-337, 2014. https://doi.org/10.1016/j.watres.2014.08.029 
SANT'ANNA, C. L.; AZEVEDO, M. T. P.; WERNER, W.R.; DOGO, C. R.; RIOS, F. R.; CARVALHO, L. R. Review of toxic species of Cyanobacteria in Brazil. Algological Studies, v. 126, p. 249-263. 2008. https://doi.org/10.1127/1864-1318/2008/0126-0251

SILVA, E. M.; GOMES; C. T.; RAMOS; C. P.; BRICIO, S. M. Ocorrência de Cianobactérias no Reservatório de Mundaú, no Estado de Pernambuco, no período de janeiro de 2010 a novembro de 2011. Vigilância Sanitária em Debate, v. 1, n. 3, p. 35-42, 2013. https://doi.org/10.3395/vd.v1i3.40

SINGER, P. C. Humic substances as precursors for potentially harmful disinfection by-products. Water Science and Technology, v. 40, n. 9, p. 25-30, 1999. https://doi.org/10.1016/S02731223(99)00636-8

SUN, Y.; WU, Q.; HU, H.; TIAN, J. Effects of operating conditions on THMs and HAAs formation during wastewater chlorination. Journal of Hazardous Materials, v. 168, p. 1290-1295, 2009. https://doi.org/10.1016/j.jhazmat.2009.03.013

UNITED STATES ENVIRONMENTAL PROTECTION AGENCY (USEPA). Drinking Water Regulations and Health Advisories. Washington, 1996, $30 \mathrm{p}$.

UNITED STATES ENVIRONMENTAL PROTECTION AGENCY (USEPA). Method 551.1. Determination of chlorination disinfection bypro- ducts, chlorinated solvents, and halogenated pesticides/herbicides in drinking water by liquid-liquid extracion and gas chromatografhy with electron-capture detection. Ohio, USA, 1995.

WILLIS, A.; CHUANG, A. W.; WOODHOUDE, J. N.; NEILAN, B. A.; BURFORD, M. A.; Intraspecific Variation in Growth, Morphology and Toxin Quotas for the Cyanobacterium, Cylindrospermopsis raciborskii. Toxicon, v. 119, n. 45, p. 307-310, 2016. https://doi. org/10.1016/j.toxicon.2016.07.005

YANG, X.; GUO, W.; SHEN, Q. Formation of disinfection byproducts from chlor (am) ination of algal organic matter. Journal of Hazardous Materials, v. 197, p. 378-388, 2011. https://doi. org/10.1016/j.jhazmat.2011.09.098

ZAMYADI, A.; CORAL, L. A.; BARBEAU, B.; DORNER, S.; LAPOLLI, F. R.; PREVÓST, M. Fate of toxic cyanobacterial genera from natural bloom events during ozonation. Water Research, v. 73, p. 204215, 2012. https://doi.org/10.1016/j.watres.2015.01.029

ZAMYADI, A.; FAN, Y.; DALY, R. I.; PREVÓST, M. Chlorination of Microcystis aeruginosa: Toxin release and oxidation, cellular chlorine demand and disinfection by-products formation. Water Research, v. 47, p. 1080-1090, 2013. https://doi.org/10.1016/j. watres.2012.11.031 